\title{
Keratinized versus non-keratinized preputial flap onlay urethroplasty: does it make any difference in the histological analysis? An experimental study in rabbits
}

Marnio Costa, Bruno Leslie, Atila Rondon, Herick Bacelar, Ricardo Mattos, Bruno Barbosa, Rosana Delcelo, Valdemar Ortiz, Antonio Macedo Jr

Division of Urology, Universidade Federal de São Paulo - UNIFESP, São Paulo, Brazil

\section{ABSTRACT}

Purpose: To compare the histological characteristics of keratinized versus non-keratinized onlay island flaps in an experimental rabbit model.

Materials and Methods: Sixteen male rabbits were randomly allocated into two experimental groups: keratinized and non-keratinized onlay island flaps. A defect was created in the ventral aspect of the penile urethra. In the keratinized group, a longitudinal island flap was harvested from the external prepuce and rotated to cover the urethral defect. In the non-keratinized group a transverse island flap was harvested from the inner prepuce. The animals were sacrificed after 2, 4, 8 and 12 weeks.

Results: The flaps were viable in all animals, and no deaths were associated with the procedure. Two urethrocutaneous fistulas were identified, one in each experimental group. A similar pattern of fibrosis was identified in both groups. The keratinized epithelium of the external prepuce kept its histological aspect and keratin production. Both keratinized and non-keratinized groups presented a slight decrease on the epithelial thickness, however without a statistically significant difference between groups.

Conclusions: In this short-term rabbit model, we observed that the stratified squamous keratinized epithelium from the external prepuce kept its keratin production. There was no statistical influence of the flap type on the mean epithelial thickness.

\section{ARTICLE INFO}

\section{Key words:}

Hypospadias; Surgical Flaps;

Foreskin; Keratins; Urethra

Int Braz J Urol. 2013; 39: 884-92

Submitted for publication:

July 27, 2012

Accepted after revision:

September 27, 2013

\section{INTRODUCTION}

Preputial and penile skin flaps remain a major tool in urethral reconstructive surgery, being used in primary or redo operations. The most commonly used flap is the classic transverse ventral island flap as described by Duckett (1) but other options are available such as a lateral penile skin flap (2) or a parameatal skin flap (3). Interestingly, despite the large amount of clinical data on flaps in hypospadias surgery, only few experimental models have been reported to allow a more in deep understanding of the healing mechanisms of this versatile technique.

Previous rabbit model study on the association of flaps and grafts for complex hypospadias surgery has been published (4). In that study the flaps were supposed to be harvested from the inner prepuce, but in one animal the flap unintentionally included a segment of external prepuce. On further histological assessment the keratinized epithelium kept its original architecture and keratin production. This anecdotal finding raised a question: Is there a difference in flaps from inner 
prepuce (non-keratinized) and flaps from the external prepuce (keratinized)? Therefore, we designed an experimental animal study to analyze the histological characteristics of keratinized versus non-keratinized onlay island flaps.

\section{MATERIALS AND METHODS}

The experimental protocol was reviewed and approved by our institution's Animal Care Committee. All handling and procedures were performed following the Brazilian Board on Animal Care guidelines. The study included 16 New Zealand white male rabbits of similar age and weight $(2-2.5 \mathrm{Kg})$. The rabbits were randomly allocated into two experimental groups: keratinized and non-keratinized onlay island flaps. A defect was created in the ventral aspect of the penile urethra. In the keratinized group, a longitudinal island flap was harvested from the external prepuce and rotated to cover the urethral defect. In the non-keratinized group a transverse island flap was harvested from the inner prepuce. The animals were sacrificed after 2, 4, 8 and 12 weeks (Figure-1).

\section{SURGICAL PROCEDURES}

Pre-anesthesia medications included midazolam $(5 \mathrm{mg} / \mathrm{Kg}$ ) followed by ketamin $(30 \mathrm{mg} /$ $\mathrm{Kg}$ ) and xilazin $(5 \mathrm{mg} / \mathrm{Kg}$ ) intramuscular. Supplemental oxygen was given through a mask. A local penile block with $2 \%$ lidocaine was administered in order to reduce general anesthetic requirements and improve analgesia in the post-operative period. All animals were operated under sterile conditions and under optical magnification (2.5X).

In all animals a standardized urethral defect was created. Initially, an 8-F catheter was placed in the urethra and then the skin web between the penis and anus was incised in order to expose the penile urethra. A segment measuring $7 \mathrm{~mm} \times 4$ $\mathrm{mm}$ from ventral wall of the middle penile urethra was excised to create a hypospadias-like defect, leaving only the dorsal "urethral plate" (Figure-2).

Once the urethral defect was created, the animals were randomly allocated into two experimental groups: keratinized (external prepuce) and non-keratinized (inner prepuce) onlay island flaps. In the non-keratinized group, an inter-

Figure 1 - Flowchart resuming the study protocol.

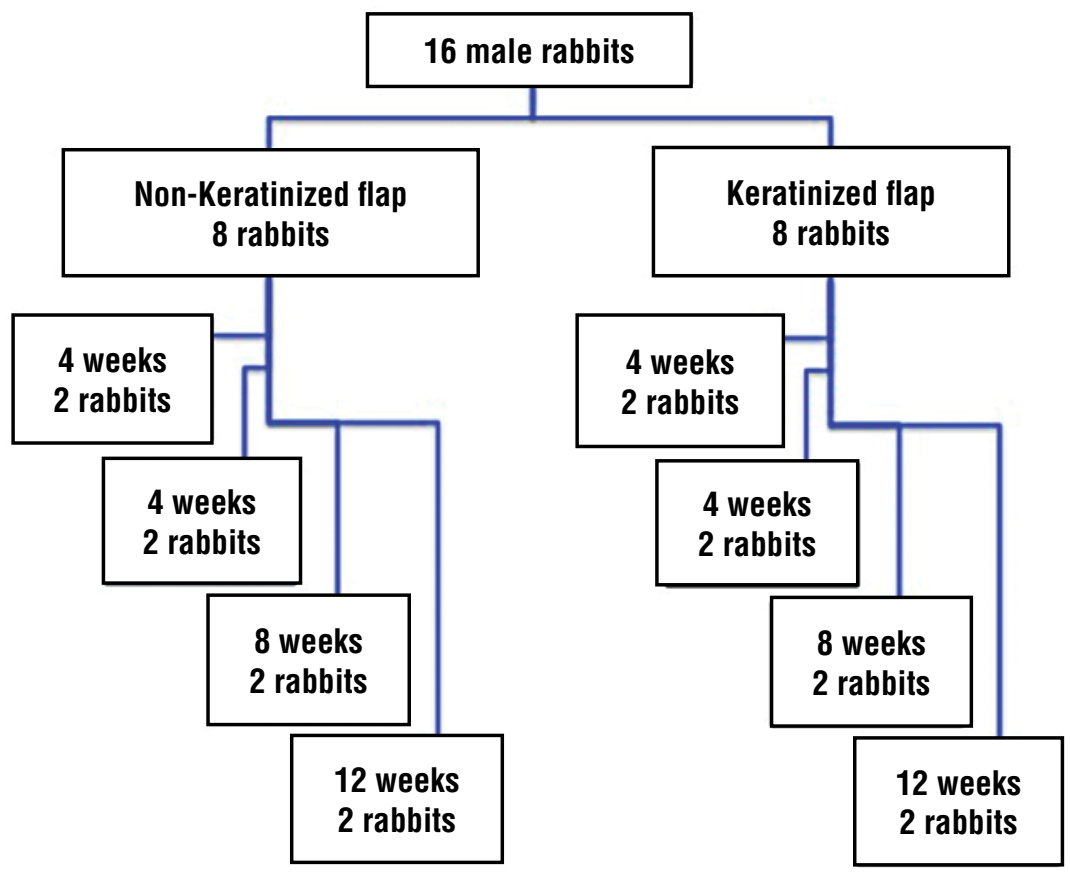


Figure 2 - A catheter was placed in the urethra and then the skin web between the penis and anus was incised in order to expose the penile urethra. A segment measuring $7 \mathrm{~mm} \times 4 \mathrm{~mm}$ from ventral wall of the middle penile urethra was excised to create a hypospadias-like defect, leaving only the dorsal "urethral plate".
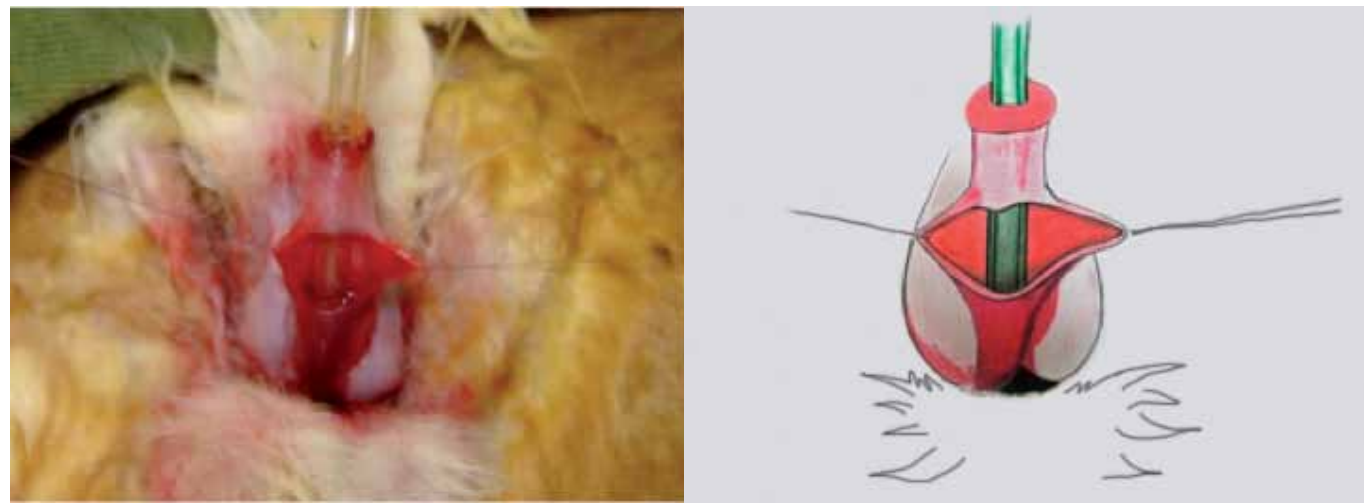

nal transverse preputial island flap was harvested similarly to a classic Duckett operation. Once mobilized (Figure-3), the flap was rotated and anastomosed with 6.0 polydioxanone in an onlay fashion to the urethral defect to cover the ante- rolateral aspect of the urethra. In the keratinized group, a longitudinal flap was harvested from the ventral aspect of the external prepuce (Figure-4), rotated and also anastomosed with 6.0 polydioxanone in an onlay fashion to the urethral defect. The

Figure 3 - Internal transverse preputial flap (A) was rotated and anastomosed with 6.0 polydioxanone in an onlay fashion to the urethral defect to cover the anterolateral aspect of the urethra (B).
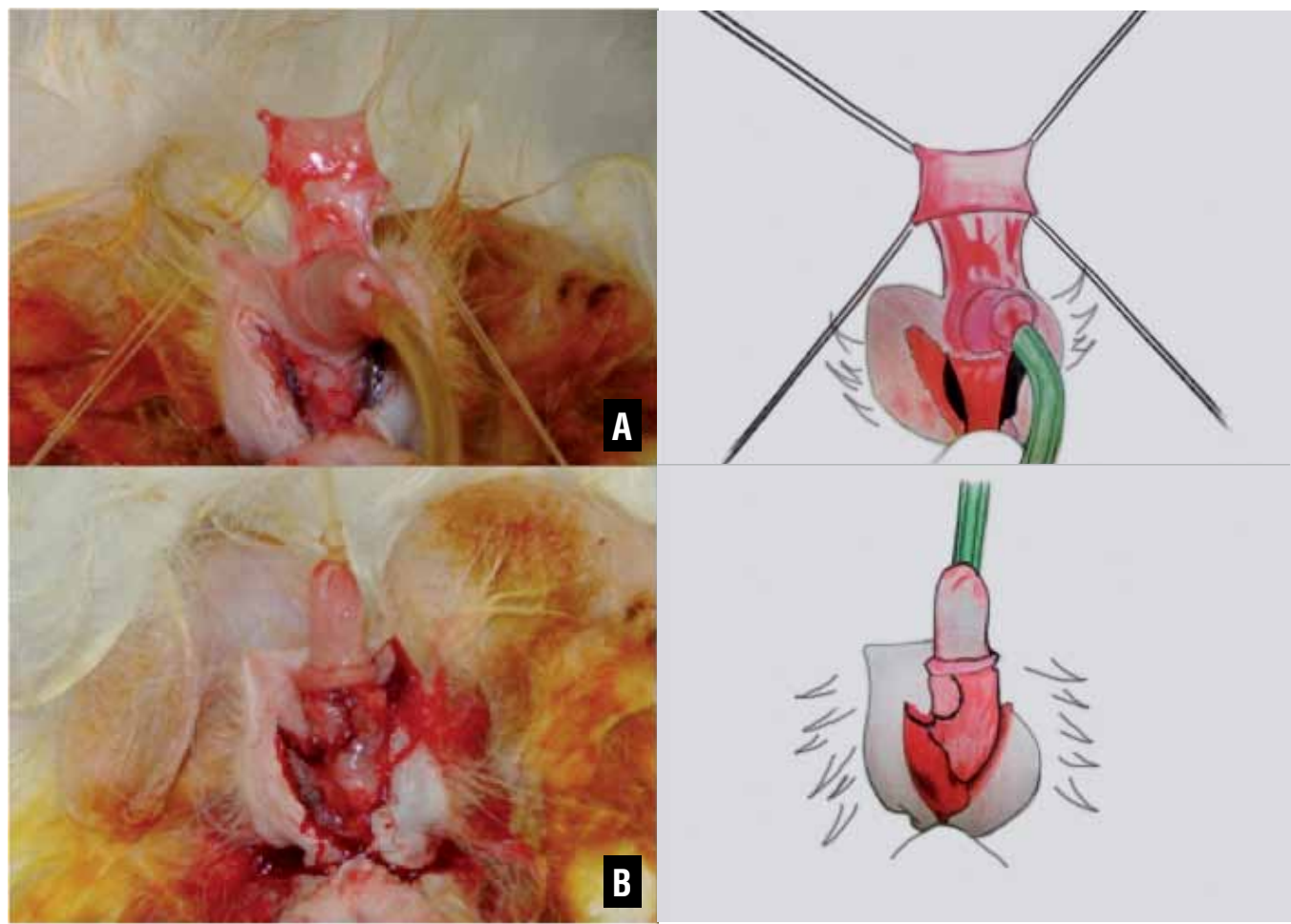
Figure 4 - In the keratinized group, a longitudinal flap was harvested from the ventral aspect of the external prepuce (A), rotated and also anastomosed with 6.0 polydioxanone in an onlay fashion to the urethral defect (B).

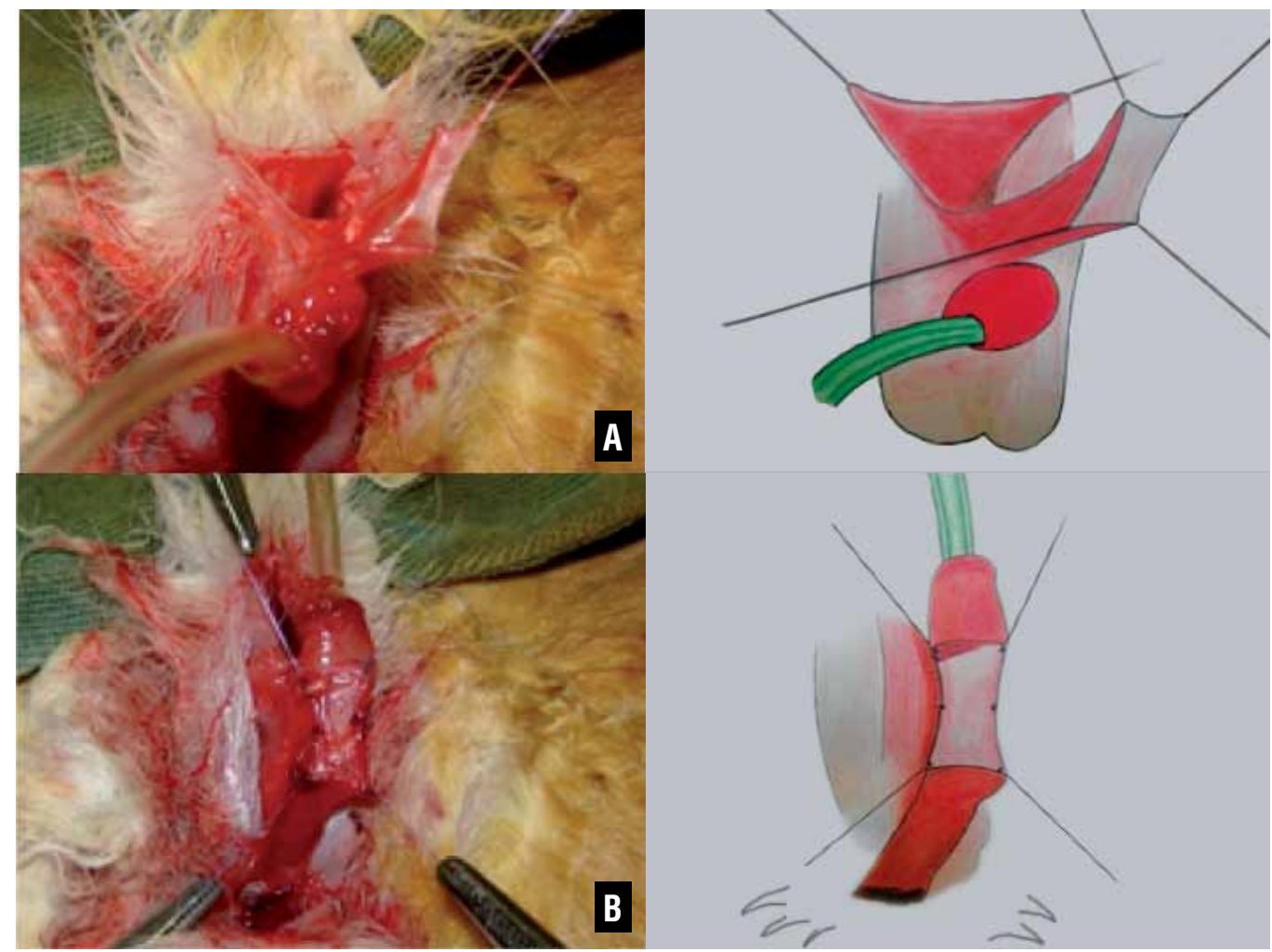

skin was closed by suturing the web between the penis and the anus. The urethral catheter was removed immediately after the procedure. No stents or dressings were used.

Two animals from each group were sacrificed at 2, 4, 8, and 12 weeks after surgery.

\section{HISTOLOGICAL PROCEDURES}

The entire penis was excised and fixed at $10 \%$ buffered formalin for 24 hours, followed by paraffin wax embedding. The pathology specimens were transversely sectioned from the glans to base of the penis. Sequential sections from the glans to the proximal urethra were provided and histological staining was performed with haematoxylin-eosin (HE), Masson's trichrome and picrosirius red.

An experienced pathologist (RD) performed histological evaluation using light microscopy in single analysis. The histological evaluation focused on the following variables: inflammation pattern, fibrosis, type of epithelial lining, epithelial thickness and possible complications. The pathologist used the same standardization of previous studies to assess the inflammatory response related to the procedure $(4,5)$. Semi-quantitative evaluation for inflammatory response included, in brief: 0, no inflammatory reaction; $1+$, minimal inflammatory reaction; 2+, moderate inflammatory reaction; $3+$, marked inflammatory reaction and; $4+$, presence of leuco-lymphocytic aggregates. The degree of sub-epithelial fibrosis was analyzed also in a semi-quantitative way, graduated from 0 to $3+: 0$, no scarring fibrosis; $1+$, minimal scarring fibrosis; $2+$, moderate scarring fibrosis and; 3 , pronounced scarring fibrosis. The following epithelial types were observed: non-keratinized stratified squamous (lining of the inner prepuce), keratinized 
stratified squamous (lining of the external prepuce) and transitional epithelium (urothelium). A quantitative analysis was performed to evaluate epithelial thickness in the area of greatest thickness near the anastomosis, through the use of a microscopic ruler (Figure-5). The following complications were considerate: fistulae, diverticulae, stenosis and dehiscence.

Mann-Whiney test was used to compare the mean values of epithelial thickness according to the type of flap. Data were collected and processed using a commercially available software package (Statistical Package for Social Sciences - SPSS, version 11.0 for Windows). A p value of less than 0.05 was considered statistically significant.

\section{RESULTS}

There were no deaths related to the procedure and all animals were sacrificed on the scheduled date. All rabbits voided spontaneously after surgery and the macroscopic appearance of the penis was normal without penile rotation. On macrosco- pic inspection, two urethrocutaneous fistulas were identified (one in the keratinized group and one in the non-keratinized group). After the sacrifice, the urethra was easily calibrated in all animals using an 8-F urethral catheter indicating that no strictures were formed. Diverticulae was identified in other two animals of non-keratinized group.

A similar pattern of inflammatory reaction was identified in both experimental groups. Two weeks post operatively, microscopic analysis showed significant polymorphonuclear cell infiltration representing an acute inflammatory reaction and an already moderate infiltration of lymphocytes and macrophages. This inflammatory profile was unchanged at 4 weeks. By the $12^{\text {th }}$ week an important decrease in the inflammatory response was identified. At $12^{\text {th }}$ week, one animal in each group developed an important lymphomonocytic reaction (Table-1).

A similar pattern of fibrosis was also identified in both groups. Moderate (2+) sub-epithelial fibrosis was observed in the groups. At $12^{\text {th }}$ week, one animal in each group developed a pronounced fibrosis (3+) (Figure-6).

Figure 5 - Microscopic ruler measuring the epithelial thickness on non-keratinized group within 8 weeks. (HE 10X)

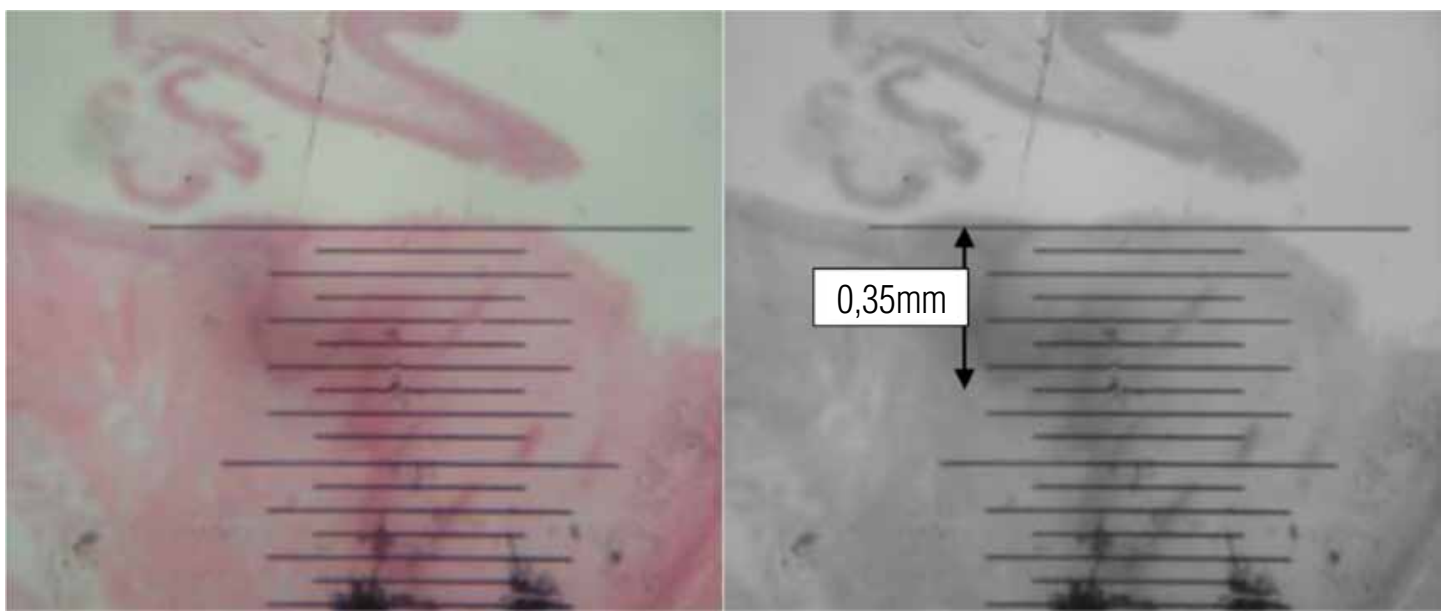

Table 1 - Inflammatory reaction.

\begin{tabular}{lcccc}
\hline Flap & 2 weeks & 4 weeks & 8 weeks & 12 weeks \\
\hline Keratinized & $2+/ 3+$ & $2+/ 3+$ & $1+/ 2+$ & $2+/ 4+$ \\
Non-keratinized & $2+/ 2+$ & $2+/ 3+$ & $1+/ 2+$ & $2+/ 4+$ \\
\hline
\end{tabular}


Figure 6 - Fibrosis: 40X Masson's trichrome (left column) and picrosirius red (right column). A and B, non-keratinized group (2 weeks); C and D, non-keratinized group (4 weeks); E and F, keratinized group (8 weeks) and; G and H, keratinized group (12 weeks).

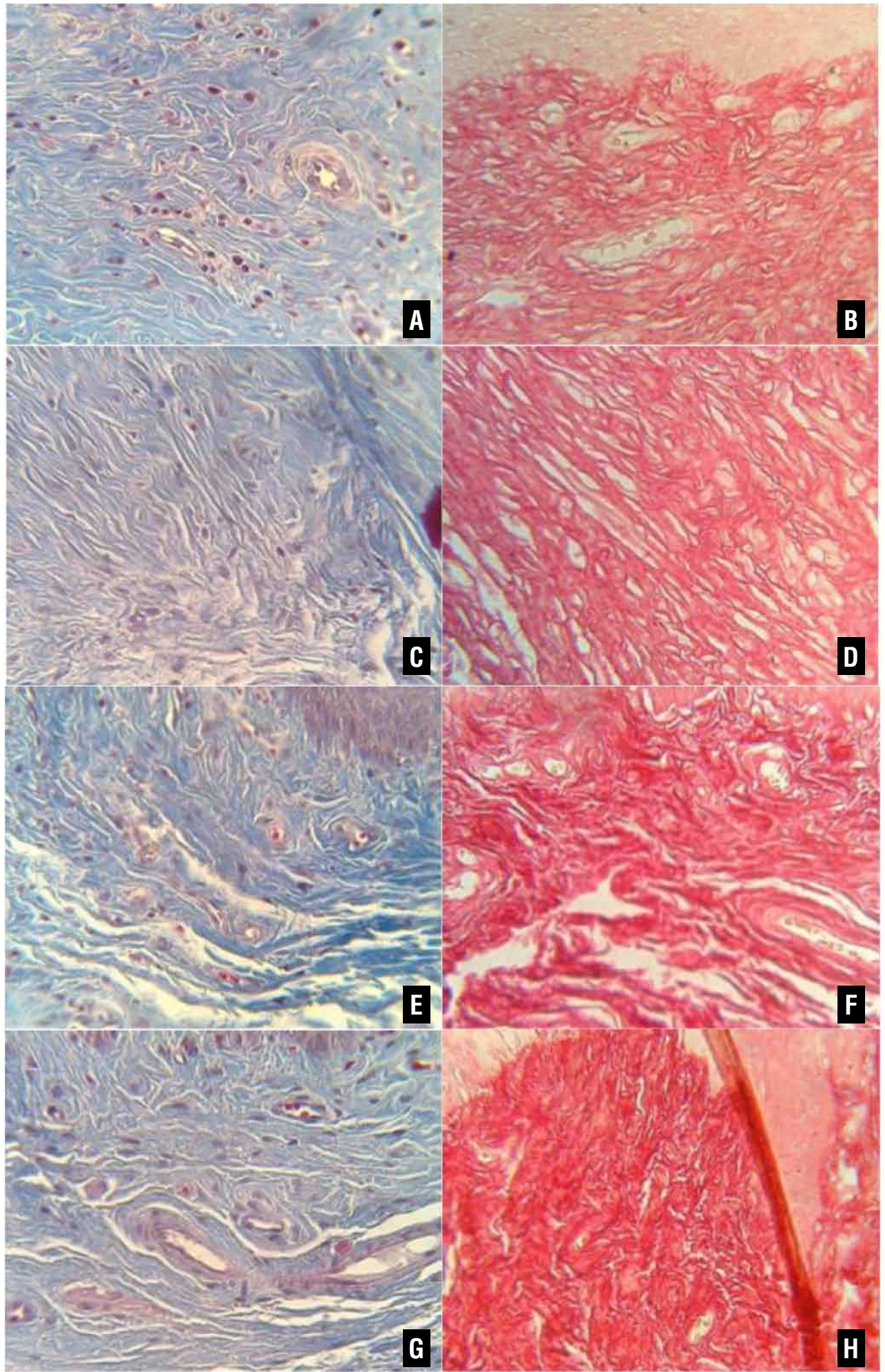


The keratinized stratified squamous lining of the external prepuce island flap kept its architecture in all time points of the study. There was no change in keratin production and the number of cell layers presented a slight decrease (Figure-7).

At the $2^{\text {nd }}$ week time point, the non-keratinized stratified squamous lining of the inner prepuce island flap kept its original microscopic appearance. The histological analysis on the 4, 8 and 12 weeks revealed a little reduction on the number of cell layers. No keratin was identified (Figure-8).
Mean epithelial thickness was $0.20 \mathrm{~mm}$ and $0.23 \mathrm{~mm}$ in the groups keratinized and non-keratinized, respectively $(\mathrm{p}=0.51)$.

\section{DISCUSSION}

Preputial island flaps are a major tool in penile reconstructive surgery, since the popularization of the method in the 80's by Duckett (1), substantial clinical data has been published (mostly retrospective clinical series). On the other hand, only

Figure 7 - Keratinized group, with a longitudinal island flap harvested from the external prepuce (HE 40X). (A) Architecture kept within 4 weeks; (B) There was no change in keratin production within 12 weeks.

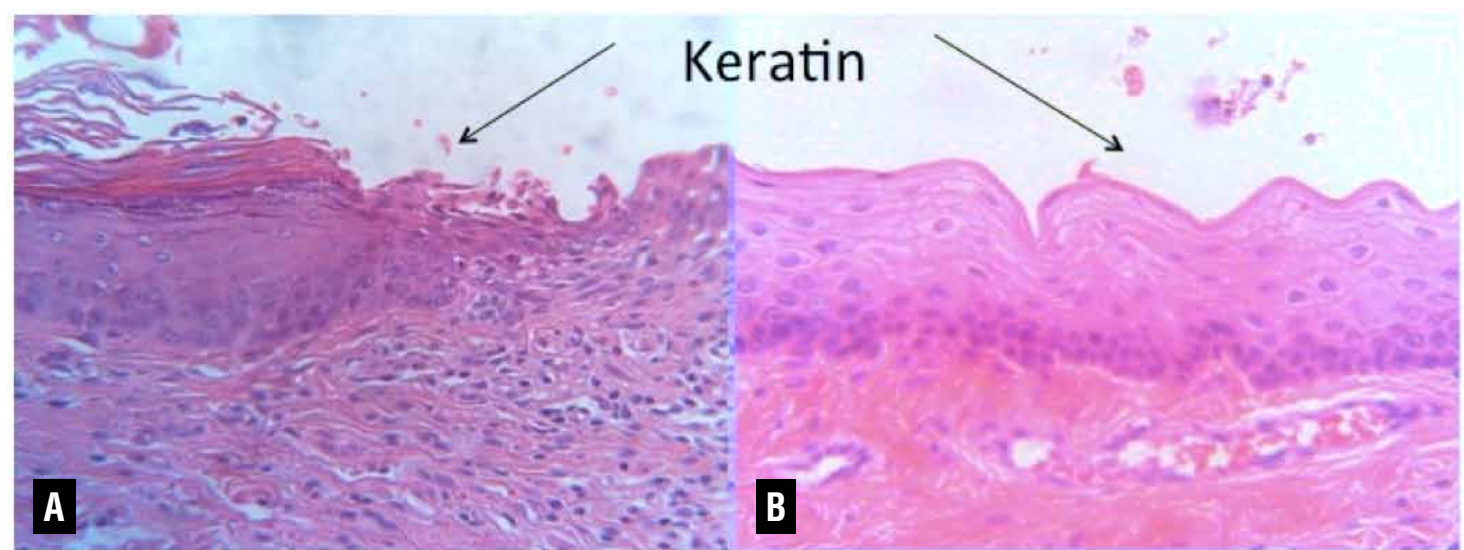

Figure 8 - Non-keratinized group, with internal transverse preputial island flap harvested similarly to a classic Duckett operation (HE 40X). (A) Histological analysis within 2 weeks revealed no keratin production; (B) Transition between the stratified squamous lining of the inner prepuce island flap and the urothelium within 4 weeks.

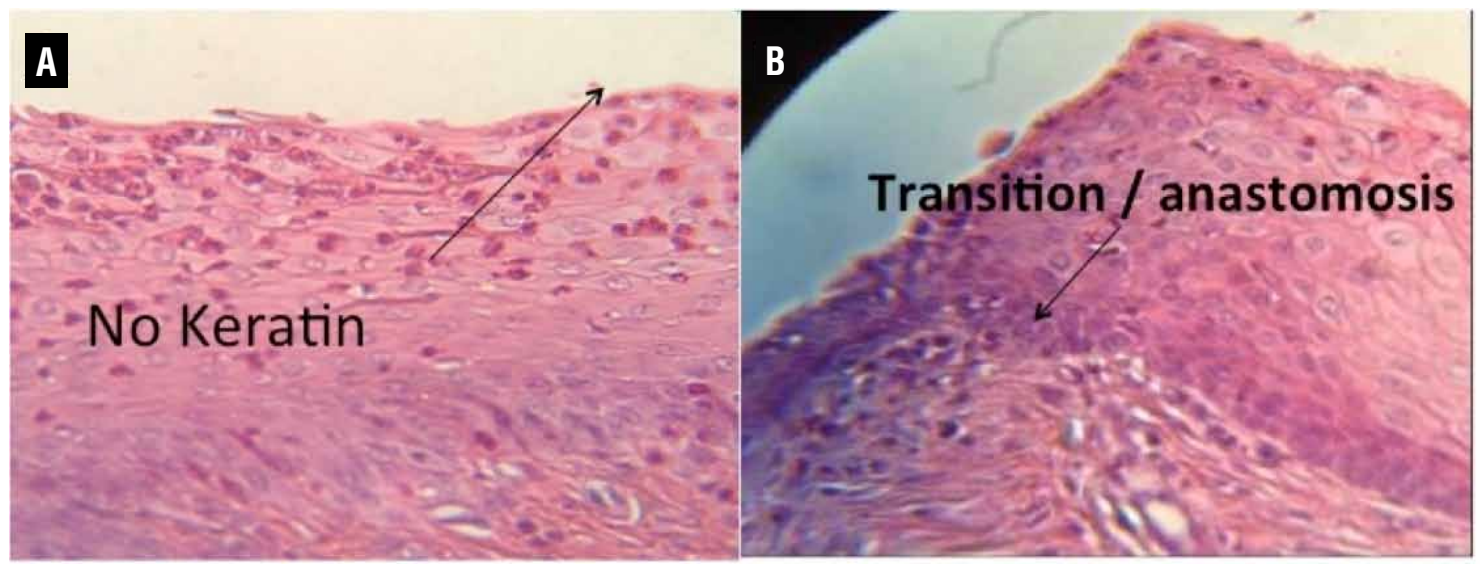


few animal models have been developed in order to allow a better understanding of the healing, tissue integration and histology of this technique. Moreover, to our knowledge, this is the first report focusing on the difference between the inner preputial (non-keratinized) and the external prepuce (keratinized) used as flaps.

Initially, the island flaps were used as tubes in one-stage operations for proximal hypospadias. Elder (5) proposed a major adaptation of the method by using the flap in onlay fashion when the urethral plate could be preserved. This modification widened the indication of the island flaps, allowing it to be used in less severe cases of hypospadias.

Scuderi (6) performed another interesting modification by harvesting the flaps in a longitudinal rectangular fashion along the vascular axis of the prepuce and rotating to ventral aspect of the penis through a "buttonhole" in the pedicle. In 2005, a long-term study of the "Scuderi procedure" (6) was reported with a reoperation rate of only $2 \%$.

The macroscopic results of our study confirm the versatility of the technique that can be reproduced even in an animal model with favorable results. Only one urethrocutaneous fistula in each group was identified. Moreover, there were no deaths related to procedure and no strictures recognized.

We also were not able to identify a different pattern of inflammatory reaction or fibrosis on the healing process when keratin was present or not. Therefore, we infer that the presence of keratin in the flap did not change the inflammatory response in this procedure.

Our most interesting findings are related to the modifications that the epithelium underwent. Leslie et al. (4) noticed in a previous study that the stratified squamous non-keratinized lining of the internal preputial island flap had a reduction in the number of cell layers and no keratin was produced in this group. On the other hand, the squamous keratinized lining of the external prepuce did not undergo a reduction in the numbers of layers with the presence of keratin production. In our findings, we noticed that keratin production remained in all animals of keratin group. Furthermore, we observed an apparent decrease in epithelial thickness in both groups, probably due to the occurrence of metaplasia. However, after statistical analysis, we verified that the difference between non-keratinized flaps mean thickness $(0.23 \mathrm{~mm})$ and keratinized flaps mean thickness $(0.20 \mathrm{~mm})$ was not significant $(\mathrm{p}=0.51)$. Therefore, there was no statistical influence of the flap type on the mean epithelial thickness.

In 1987, Talja (7) developed a similar experimental rabbit model for preputial grafts, although the author did not specify if preputial graft was keratinized. The results suggested the same by demonstrating that the preputial graft did not undergo modifications and kept the same number of cell layers in a rabbit model.

Another finding that corroborates with the hypothesis that the keratinized epithelium keeps its architecture and keratin production when applied as a flap to the urethra, is the report by Leger (8). The author performed endoscopic biopsies after series of cutaneous urethroplasties, demonstrating that the epidermis still showed a keratin malphigian layer, often dense and with hyperacanthosis, hair and sebaceous glands.

We then theorize that the keratin layer in the external prepuce may act as a barrier, preventing the interaction between the urine and the epithelium itself and further modifications in the architecture. We observed lower complication rate in the keratinized group, particularly diverticula.

Our study has important limitations, the most salient being the short-term of experiment. It is not clear that our findings will last in the long-term. Another relevant one is related to the small number of animals in each group, not allowing us to conclude if the clinical outcome (i.e. fistulas and diverticulae) would be different in the groups. Furthermore, future studies can better exploit histological and quantification analysis performing other tests as Proliferating Cell Nuclear Antigen (PCNA), the occurrence of apoptosis (Tunel) and nuclear density.

Despite these limitations, we believe there is value in the current report. By adding to the limited literature on the topic, we provided additional experimental data on the complex process of healing and integration of flaps in urethral surgery showing that keratinized and non-kera- 
tinized epithelium undergo different histological modifications when used in urethroplasty.

\section{CONCLUSIONS}

In this short-term rabbit model, we observed that the stratified squamous keratinized epithelium from the external prepuce kept its keratin production. Both keratinized and non-keratinized groups presented a slight decrease on the epithelial thickness, however without a statistically significant difference between groups.

\section{CONFLICT OF INTEREST}

None declared.

\section{REFERENCES}

1. Duckett JW: The island flap technique for hypospadias repair. Urol Clin North Am. 1981; 8: 503-11.

2. el-Kasaby AW, el-Beialy H, el-Halaby R, Nowier A, Maged A: Urethroplasty using transverse penile island flap for hypospadias. J Urol. 1986; 136: 643-4.

3. Koyanagi T, Nonomura K, Gotoh T, Nakanishi S, Kakizaki H: One-stage repair of perineal hypospadias and scrotal transposition. Eur Urol. 1984; 10: 364-7.

4. Leslie B, Barboza LL, Souza PO, Silva PS, Delcelo R, Ortiz $V$, et al.: Dorsal tunica vaginalis graft plus onlay preputial island flap urethroplasty: experimental study in rabbits. $J$ Pediatr Urol. 2009; 5: 93-9.
5. Elder JS, Duckett JW, Snyder HM: Onlay island flap in the repair of mid and distal penile hypospadias without chordee. J Urol. 1987; 138: 376-9.

6. Scuderi N, Chiummariello S, De Gado F: Correction of hypospadias with a vertical preputial island flap: a 23-year experience. J Urol. 2006; 175: 1083-7; discussion 1087.

7. Talja M, Kivisaari L, Mäkinen J, Lehtonen T: Free tunica vaginalis patch in urethroplasty. An experimental study. Eur Urol. 1987; 13: 259-63.

8. Leger P: Surveillance endoscopique des urétroplasties cutanées. Journées urologiques de Necker. 1983; pp. 12-7.

Correspondence address: Atila Rondon, MD

Rua Maestro Cardim, 560 / 215

São Paulo, SP, 01323-000, Brazil

Fax: +55 11 3287-3954

Email: atilarondon@hotmail.com 